\title{
DEXA Contribution in Bone Densitometry Evaluation on Osteoporosis in Senegal: Results of a Study Realized in African Black Populations
}

\author{
Ndèye Marième Diagne-Gueye, Thierno Oumar Soko, Bineta Ndiaye, \\ Ismaïla Diedhiou, Abdou-Rajack Ndiaye \\ Service de Médecine Polyvalente, Services Médicaux, Hôpital Principal de Dakar, Dakar, Sénégal \\ Email: abdourajack@yahoo.fr
}

How to cite this paper: Diagne-Gueye, N.M., Soko, T.O., Ndiaye, B., Diedhiou, I. and Ndiaye, A.-R. (2016) DEXA Contribution in Bone Densitometry Evaluation on Osteoporosis in Senegal: Results of a Study Realized in African Black Populations. Open Journal of Rheumatology and $\mathrm{Au}$ toimmune Diseases, 6, 85-95.

http://dx.doi.org/10.4236/ojra.2016.64014

Received: August 12, 2016

Accepted: November 1, 2016

Published: November 4, 2016

Copyright (c) 2016 by authors and Scientific Research Publishing Inc.

This work is licensed under the Creative Commons Attribution International License (CC BY 4.0).

http://creativecommons.org/licenses/by/4.0/

\begin{abstract}
Osteoporosis, a public health problem is able to occur severe fractures and its prevalence increases in developing countries. Several factors involved in its pathogenesis. These factors are: Heredity, elderly, race, ethnicity, hormones, physical inactivity, sunlight exposure, vitamin D deficiency and certain drugs. In Africa, few data are available and the disease prevalence is maybe underestimated. In Senegal, measure of bone mineral density is recent. In this study, our objective was to determine DEXA patients profile and epidemiological, clinical characteristics of patients for densitometry examination. Thus, 43 cases were collected; lumbar and femoral sites were evaluated. Median lumbar T-score was 0.91 and the femoral T-score was 0.16 . Osteopenia was found in 35\% of patients and osteoporosis in 26\%. Bone Mineral Density was abnormal in $60 \%$ of cases. This study is another proof of documented reality of osteoporosis in Senegal. It determines frequency in a population at risk. For the future, it opens way for further larger studies to get better profile of the patients and prevalence osteoporosis in Senegal.
\end{abstract}

\section{Keywords}

Osteoporosis, Bone, DEXA, Africa, Black Populations

\section{Introduction}

Osteoporosis was defined in 1993 as a "disease characterized by low bone mass and micro architectural deterioration of bone tissue, leading to enhanced bone fragility and a consequent increase in fracture risk". A more recent definition from the NIH Consensus. 
Development Panel on Osteoporosis defines osteoporosis as a skeletal disorder characterized by compromised bone strength predisposing a person to an increased risk of fracture. Clinically, bone strength is estimated by non-invasive assessment of bone mineral density (BMD) by dual-energy X-ray absorptiometry (DXA). Numerous epidemiologic studies confirm that low BMD is among the strongest risk factors for fracture. As endorsed by the World Health Organization (WHO), the clinical diagnosis of osteoporosis is based on BMD measurements and the presence of fractures. Osteoporosis is the most common systemic bone disease osteopathy [1]. It is responsible for multiple fractures and can be severe. This is a major public health problem [2]. Also, its prevalence is increasing due to the increase in life expectancy. Over the past two decades, the management of osteoporosis has benefited from a better understanding of the pathogenic mechanisms, diagnosis and treatment advances. The pathology result of several factors often entangled and to varying degrees. These factors include heredity, advanced age, race, ethnicity, hormones, physical inactivity, exposure to sunlight, vitamin D deficiency in calcium intake and taking certain medications [3]. Demographically, it is established that the mode of living is different from one continent to another. [4] Add to this bone mass; is considered higher in the black population. In addition, many developing countries have a lower life expectancy. These elements are likely to estimate the prevalence of osteoporosis as relatively low in the continent [3] [5]. However, these regional differences deserve to be further clarified and better explained. In Senegal, the feasibility of measuring bone mineral density is recent, and is essentially in private structures. Thus, we conducted a study to determine the profile and ostéodensitométrique epidemiological and clinical characteristics of the patients received in a private unit of Dakar.

\section{Patients and Methods}

\subsection{Framework}

This study was conducted in private medical imaging called IMODSEN Centre (Modern imaging of Senegal). This center is a medical complex at Costal Road in Dakar. The center aims to provide and develop a modern medical imaging. It has a 64-slice scanner, a new generation MRI, and digital radiology table, digital mammography, dental panoramic X-ray, a color Doppler ultrasound and an apparatus digital densitometry.

\subsection{Type and Period of Study}

Our study is a retrospective descriptive type. It was conducted over a period of six months from 1 January 2014 to 30 June 2014. It focused on the epidemiological, clinical and outcome measurements of bone mineral density by dual photon bone densitometry unit at X-ray All patients were referred for this examination at IMODSEN center. It was a non-interventional study. No additional examination or therapeutic intervention was made in patients. 


\subsection{Inclusion Criteria}

The records included were those of patients who have benefited from the realization of a BMD at the Centre for Imaging after being sent to measure bone mineral density. Prior to the agreement of each patient was obtained through a standard electronic form to integrate the study.

\subsection{Exclusion Criteria}

Inoperable cases due to incomplete data were not included.

\subsection{Data Collections and Exploitation Method}

Data were collected on survey sheet. For each patient a patient record were retained such as a questionnaire data and the results of the results of the ostéodensitométrique examination. Thus were collected: the data of the civil status of patients, the examination pattern, age of menopause, race and ethnic origin, personal and family history of fracture, and notions of taking the long course corticosteroids, prolonged immobilization, the existence of a smoking or alcohol consumption and regular supply of dairy products. All data collected on data sheet were listed on Microsoft Excel Spreadsheet Version 2010. Statistical calculations were performed by the SPSS software.

\section{Results}

\subsection{Workforce Ages and Sex Distribution}

After the initial work, 46 files were collected. The files were included among 43 and 3 were excluded because they were incomplete and unusable for lack of densitometry results. The average age of patients was 60.47 years and the median age of 59.5 years, ranging 32 to 79 years. According to the age's data collection, 33 were 50 years or older, representing $91.67 \%$. While 3 patients were aged under 50 , or $8.33 \%$ of patients. The gender breakdown is as follows: 40 (93.02\%) women and 03 men $(6.98 \%)$. Which constitutes a sex ratio of 0.75 . Some patients had indeterminate results, or missing data because they have been lost of view or their files were lost or incompletely written.

\subsection{Age of Menopause}

Upon completion of the bone densitometry examination, the average duration of menopause was 12.37 years, while the median was 11 years with the following extremes: minimum 2 years and maximum 31 years.

\subsection{Racial and Ethnic Origin}

Among the 43 patients examined in bone densitometry, 29 were black and 14 were white race (13 patients) or mixed (1 patient). On Table 1, are shown frequencies and percentages of patients by their country origin.

\subsection{Reasons for Osteodensitometry Examination}

Among the 43 total cases, 26 requests were motivated BMD. Seventeen applications 
Table 1. Frequencies and percentages of patients by origin country.

\begin{tabular}{ccc}
\hline Origins & Frequencies & Percentage \\
\hline Mali & 2 & 4.76 \\
Benin & 1 & 2.38 \\
Cap Vert & 1 & 2.38 \\
France & 6 & 14.29 \\
Lebanon & 6 & 14.29 \\
Guinea & 1 & 2.38 \\
Niger & 2 & 4.76 \\
USA & 1 & 2.38 \\
Senegal & 22 & 52.38 \\
\hline
\end{tabular}

were not written motivations. The medical reasons of osteodensitometry and their respective frequencies are presented in Table 2.

\subsection{Fracture History}

A personal history of fracture was found in 15 patients or $34.88 \%$ of cases. While 28 patients (65.12\% of cases) showed no personal history of fracture. A family history of fracture was described in 12 patients (28.57\% of cases) when 30 patients reported no family history of fracture. They represented $71.43 \%$ of the patients examined. So out of the 43 patients, 22 had at least one existing fracture, whether personal or family. They represented $52 \%$ of patients. By combining the two types of history, in 20 cases ( $48 \%$ of patients) any existing fracture was found.

\subsection{Prolonged Systemic Corticosteroid Therapy}

A decision on long-term systemic corticosteroids was found in 2 patients (5\% of cases), whereas 38 patients (95\% of cases) no concept of steroids was found in the interrogation. It is noteworthy that in 3 patients, a precise answer has been obtained to the question about the existence or not of prolonged systemic corticosteroid therapy.

\subsection{Prolonged Immobilization}

Among the 41 responses to the survey forms, a notion of prolonged immobilization was found in 12 cases (29.27\% of patients who responded to the question). While in 29 patients $(70.73 \%$ of cases $)$ the examination had not found the notion of prolonged immobilization.

\subsection{Tobacco and Alcohol}

37 patients reported no concept of smoking, they represented $88 \%$ of cases. A smoking case was found in 5 patients examined, 12\% of cases. In 2 cases, a precise answer to this question was not obtained. As regards the alcohol, it is present in 3 cases (7\% of pa- 
Table 2. Medical reasons of osteodensitometry and their respective frequencies.

\begin{tabular}{|c|c|c|c|c|}
\hline & Frequency & Percentage & $\begin{array}{l}\text { Cumulated } \\
\text { frequency }\end{array}$ & $\begin{array}{l}\text { Cumulated } \\
\text { percentage }\end{array}$ \\
\hline Osteoporosis evaluation & 1 & 3.85 & 1 & 3.85 \\
\hline Osteogenesis evaluation & 1 & 3.85 & 2 & 7.69 \\
\hline Bone Mineral Density measure & 1 & 3.85 & 3 & 11.54 \\
\hline $\begin{array}{l}\text { Diffuse bone demineralization } \\
\text { and abnormal spine }\end{array}$ & 1 & 3.85 & 4 & 15.38 \\
\hline Locale bone demineralization & 1 & 3.85 & 5 & 19.23 \\
\hline Diffuse bone pains & 1 & 3.85 & 6 & 23.08 \\
\hline Post traumatic fracture & 1 & 3.85 & 7 & 26.92 \\
\hline Knee pains & 1 & 3.85 & 8 & 30.77 \\
\hline Lumbar pains and bone destruction & 1 & 3.85 & 9 & 34.62 \\
\hline Lumbar pains and osteoporosis suspicion & 1 & 3.85 & 10 & 38.46 \\
\hline Menopausal evaluation & 1 & 3.85 & 11 & 42.31 \\
\hline Menopause at 50 years & 1 & 3.85 & 12 & 46.15 \\
\hline Early menopause & 1 & 3.85 & 13 & 50.00 \\
\hline $\begin{array}{c}\text { Menopause with hormone substitution } \\
\text { treatment }\end{array}$ & 2 & 7.69 & 15 & 57.69 \\
\hline Osteoporosis & 3 & 11.54 & 18 & 69.23 \\
\hline Osteoporosis and back pains & 1 & 3.85 & 19 & 73.08 \\
\hline Osteoporosis suspicion & 2 & 7.69 & 21 & 80.77 \\
\hline Ovarian Tumor & 1 & 3.85 & 22 & 84.62 \\
\hline Vertebral Fracture & 1 & 3.85 & 23 & 88.46 \\
\hline Basal measurement & 3 & 11.54 & 26 & 100.00 \\
\hline
\end{tabular}

tients). Furthermore, the alcohol was absent in $93 \%$ of the cases among 40 other patients.

\subsection{Consumption of Dairy Products}

In our series, 28 patients (65.12\% of cases) reported having a regular consumption of milk products. In dairy consumption was not quantified in this study in calories. However this notion of regular consumption of dairy products was absent in 15 patients (34.88\% of cases).

\subsection{Bone Densitometry}

In this study, 43 patients had actually received a measure of bone mineral density. The Figure 1 shows global results of bone densitometry examination among all 43 patients.

This measurement was performed on the lumbar spine and the femoral site. Thus the average lumbar $\mathrm{T}$-score was -0.91 and median -1.00 with standard deviation 2.00 . The 


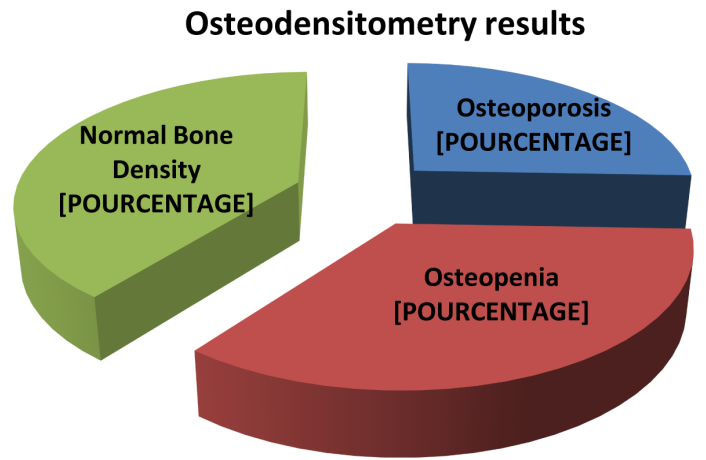

Figure 1. Global results of bone densitometry examination of 43 patients.

extremes of this lumbar T-score were as follows: minimum-maximum of 5.20 and 3.80. In the femur, the T-score measured in patients average was-and a median of $0.16-0.10$ with a standard deviation of 1.31. The minimum femoral T-score founded in patients was-2.70, and the maximum value of this T-Score femoral was 3.10. Bone mineral density at the wrist site was not measured in our patients. After the measurement, bone mineral density was abnormal in 26 patients (60\% of cases). 17 patients had normal Bone Mineral Density (39\%). Among patients with abnormal BMD, there was 15 cases (35\%) of osteopenia (T-score $\leq-1 \leq-2.5$ ) and 11 cases (26\%) with osteoporosis (T-score $\leq-2.5$ ). On the Figure 2, we have panoramic distribution of patients by lumbar T-Score lombaire in Normal zone, and Osteopenia or Osteoporosis areas. FIG exposes the distribution of different patients on normal BMD fields, osteopenia and osteoporosis in function of the measure of their lumbar T-Score. It appears from these results that $60 \%$ of patients referred for bone densitometry, have either osteoporosis or osteopenia documented.

\section{Comments}

Osteoporosis is the result of several factors involved to various degrees. As we emphasized in the introduction, heredity, age, race, ethnicity, hormonal production, inactivity, sun exposure, the vitamin D deficiency, calcium, as well that certain drug taken. Thus, from one country to another or from one continent to another, epidemiological and clinical characteristics of osteoporosis vary. In Black Africa the number of studies on the pathology remains insufficient to describe in detail the characteristics of this particular epidemiological and clinical. Our study is preliminary, however, it certainly gives an idea of the profile of patients that are referred for measuring bone mineral density in a Dakar center. Indeed, it helped to bring a first response to important questions about the patient's age, the distribution of the disease by sex, the clinical profile of patients and their result after ostéodensitométrique examination.

\subsection{Number, Age and Sex Distribution}

With the aging population, osteoporosis is becoming a very common condition worldwide, especially in women after menopause. In our study, this frequency is confirmed. 


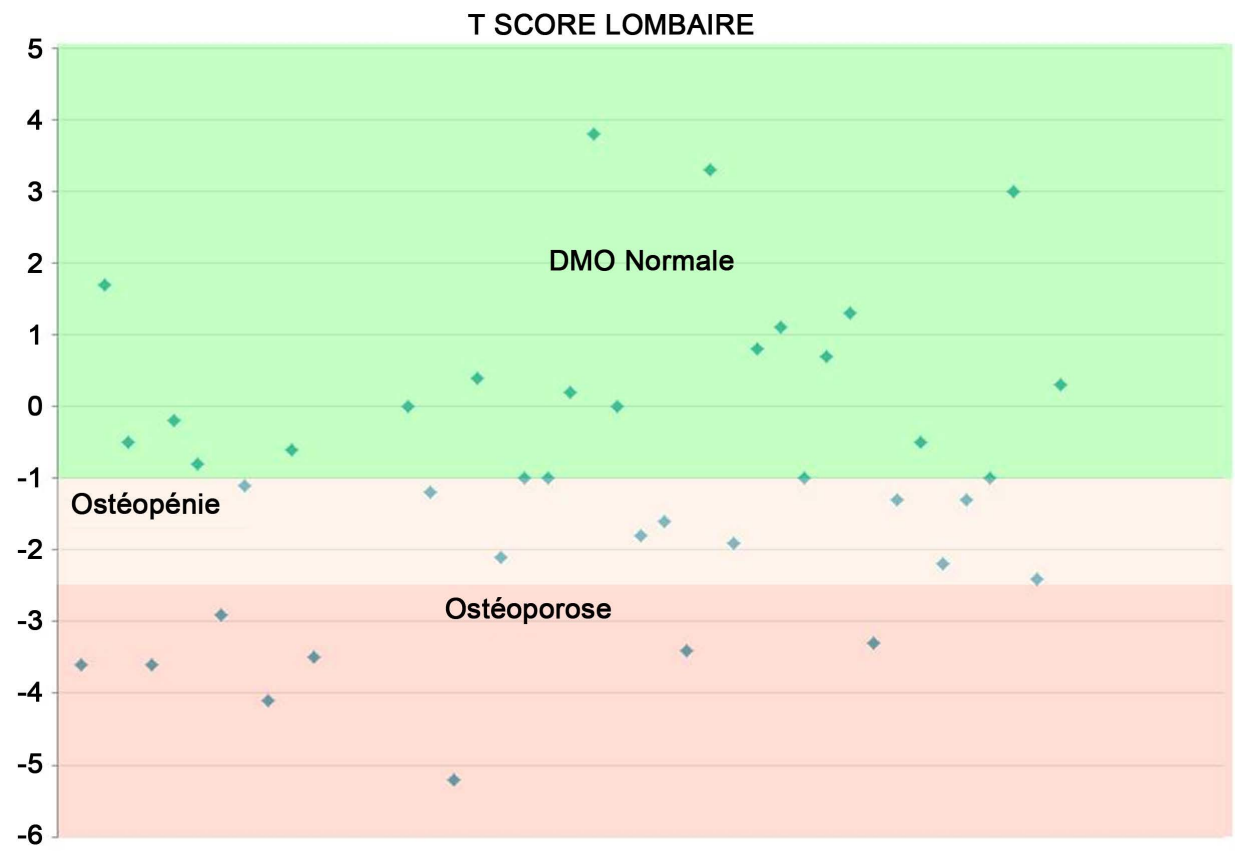

Figure 2. Panoramic distribution of patients by lumbar T-Score lombaire in Normal zone, and Osteopenia or Osteoporosis areas. Osteopenia: 35\%-Osteoporosis: 26\%-Normal Bone Density: $39 \%$.

In fact 17 of the 43 patients examined had osteopenia or osteoporosis. This frequent is important especially as it relates to $39 \%$ of the files we collected. These results confirm the recent work that consider osteoporosis as a major public health problem with a frequency of $39 \%$ in women 65 years and older. The same study estimated that $70 \%$ the frequency of the disease in patients aged 80 and over [6]. The condition affects as expected, seniors sixties. But it can affect young adults as shown in the minimum age of 32 in our series. At the same time we find that our patients are relatively younger than those of the Western series. In fact the oldest of our patients was 79 years old. In the European series the mean age of patients is estimated at 78.4 years with extremes of 64 and 100 years [7]. This difference could be explained by the greater aging of European populations and relatively lower life expectancy in sub-Saharan Africa. Concerning the distribution by sex, the female remains constant with $93.02 \%$ women against $6.98 \%$ men in our study. These results support the role of menopause in the mechanisms of osteoporosis should be remembered that the concerns a bone without estrogens. Also, it should be stressed that the occurrence of at least osteopenia is early because it appears at the end of the first decade postmenopausal (median age of menopause 12 years old).

\subsection{Racial or Ethnic Factor}

The presence of a majority of black patients confirms that the disease affects indeed our people but the racial predominance is explained by the fact that the study was performed in a so black African Senegalese population. The small sample size did not allow to draw a conclusion as to whether or not predominantly by ethnic group. 


\subsection{Indications of Bone Densitometry}

The indications of bone densitometry are numerous and vary from one patient to another. Personal or family history of fractures are most commonly found. These personal history were found in $65 \%$ of patients and family in $28 \%$ of cases [3] [8]. It must be remembered that: whatever the indication, bone densitometry is only useful if the pathological outcome of the review resulted in a patient's treatment decision. The main indications are: history of fracture called low-energy device, no traumatic vertebral fracture or obvious tumor, endocrine disease that can induce osteoporosis (untreated hyperthyroidism, hyper blood cortisol, hypogonadism, and primary hyperparathyroidism), long-term use inducing osteoporosis drugs or orchiectomy. Postmenopausal women is particularly at risk. That is why it has the following additional information: a family history of low energy fractures of the femoral neck, a body mass index less than $19 \mathrm{~kg} / \mathrm{m}^{2}$ and therefore early menopause before age 40 [9].

\subsection{Long-Term Systemic Corticosteroids}

The long-term systemic corticosteroids was rarely found because only $5 \%$ of patients had reported prolonged glucocorticoid therapy. These figures seem low but can be explained by the fact that patients were followed by specialists who would have a better mastery of the rules of prescription steroids for long periods. A larger study could best build on these results. This statement also applies to prolonged immobilization, which in contrast seems present in significant proportions. Nevertheless, this anamnestic data was found in 12 cases. Prolonged immobilization concept deserves to be better specified above its duration quantified in subsequent studies.

\subsection{Alcohol or Tobacco Consumption}

In our study, the proportions of patient's consumers of tobacco or alcohol are low. Only 5 patients were smokers and 3 occasionally drank alcohol. The majority of patients were neither alcohol nor tobacco consumers. These results lower than those described in Western studies, can be explained by cultural, behavioral and lifestyle and dietary differences between African and European black older populations. For example, in England $16 \%$ of people over 65 smoke; which represents about 1.5 million people and estimated in 2020, their number will reach 2 million smokers [10].

\subsection{Consumption of Dairy Products}

The dietary intake of dairy products do not seem sufficient because they do not cover all of our patients. But with no exact quantification tool of dairy consumption, the impact on the accession or not an osteoporosis or osteopenia cannot be clearly established. Therefore, it seems important to work towards the establishment of a multidisciplinary team of a measurement tool indicative of calcium intake and taking into account the food habits in our regions. It is also important to note that this deficit is not easy to fill. In a French study, it is reported that the average dietary calcium intake among postmenopausal women, was also well below the amounts recommended by the 
national guidelines [11].

\subsection{Bone Densitometry}

This means that on average, our patients are osteopenia particularly femoral. The upper end of the femur is the seat of the severity of the pathology that required fracture risk and consequences of these fractures on both morbidity plan in mortality. The morbidity and mortality is about $30 \%$ a year in fracture. Some patients have T-Score particularly lowered that show the evidence of severe osteoporosis until proven otherwise. These lower scores are illustrated by the minima of -5.20 and -2.70 respectively at the lumbar spine and femoral site. Thirty-five percent of our patients had osteopenia and $26 \%$ of them had osteoporosis. This trend confirms a large, recent study by Tunisian Ben Abdelghani. This study included 368 women aged between 45 and 80 years with a mean age of 51.8 years and had the following results: $32.2 \% 10.1 \%$ osteopenia and osteoporosis [12]. Our frequencies are relatively higher, this may be related to the fact that they have measured bone densitometry ultrasound which is less efficient than the dual-energy X-ray absorptiometry we used [13] [14] [15]. Our 11 patients with osteoporosis (26\%) are in most fracture risk zone and require the initiation of therapeutic measures. This allows that after bone densitometry at least 1 in 4 is an indication of anti-osteoporotic treatment and that treatment can be started in order to prevent fracture. Our study has enabled to highlight Bone Mineral Density abnormal in 26 patients. So $60 \%$ of patients referred for bone densitometry, had either osteopenia or established osteoporosis. This frequency is high because for the majority of patients received. But there is a frequency found in patients who had a clinical or para clinical suspect osteoporosis. This suggests that when bone densitometry is requested before a strong suspicion in over half the cases bone mineral density is abnormally reduced as described so well the summary graph of the results. This is a sensitive examination and a considerable contribution to our practice because he affirms the existence of osteopathy.

\section{Conclusion}

Osteoporosis remains a public health problem. It is responsible for many serious fractures. The fracture of the proximal femur is the most severe form. Data on the disease are rare in black Africa. So we conducted this study in Senegal in order to determine the ostéodensitométrique epidemiological profile and clinical characteristics of our patients. This cross-sectional descriptive study (one of the first in Senegal) was conducted using the collected files from IMODSEN Centre. In our study, 43 patients had a BMD at lumbar and femoral sites. The average lumbar T-score was 0.91 and the femoral T-score was 0.16 . Osteopenia was found in $35 \%$ of patients and osteoporosis in $26 \%$ of osteoporosis. Our study therefore was possible to objectify a pathological BMD in 60\% of cases. This study has the merit of confirming the reality of osteoporosis in Senegal and indicates the proportion of disease in a population at risk. It opens the way for further multicenter studies larger to get a better idea of the profile of the patients and the magnitude of the osteopathy in Senegal. It simultaneously allows validating the results 
obtained in this Centre for Imaging by comparing them to those in the medical literature. But one of the limitations of this study consists of the small sample size, the lack of precision in some measures, including calcium intake and physical activity. At present the most important advocacy is to improve access to anti osteoporotic treatment once the diagnosis to avoid serious fracture occurrence that threatens the vital prognosis of the patient. The second plea is to educate practitioners to better knowledge of indications of bone densitometry and its place in the diagnosis of osteoporosis.

\section{Acknowledgements}

Professor Abdourahmane NIANG, head Pulmonology Clinic of Dakar Military Teaching Hospital and all staff and team members of Dakar Medical Imaging Office IMODSEN

\section{Ethical Clearance}

Authors declare that they have ethical clearance of this study.

\section{Conflict of Interest}

Authors declare that no one of them had or have a conflict of interest in this study.

\section{References}

[1] Chappard, D. and Chapurlat, R.D. (2009) Osteoporosis, Better Knowledge for Better Treatment. Rueil-Malmaison: Wolters Kluwer: Lippincott Williams \& Wilkins.

[2] El Maghraoui, A. (2013) Malnutrition, Wasting and Osteoporosis. Second Bone Fragilities, 80, 100-104.

[3] Berthélémy, S. (2014) Osteoporosis, a Skeletal Disorder. Actualités Pharmaceutiques, 53, 48-52. http://dx.doi.org/10.1016/j.actpha.2014.08.011

[4] Hassani, M.A. and Ndongo, S. (2011) Ostéodensitométrique Status of 102 Patients Received the First Bone Densitometry unit Geriatrics Center Ouakam. Medical Doctor, Cheikh Anta Diop in Dakar, Dakar.

[5] El Maghraoui, A. (2004) Osteoporosis and Ankylosing Spondylitis. Revue du Rhumatisme, 71, 573-578. http://dx.doi.org/10.1016/j.rhum.2003.06.002

[6] El-Maghraoui, A. (2010) Osteoporosis. Imp. El-maarif al-Jadida, Rabat.

[7] Silva, B.C., Costa, A.G., Cusano, N.E. and Bilezikian, J.P. (2013) Osteoporosis: What's New and on the Horizon. Clinical Obstetrics and Gynecology, 56, 730-738. http://dx.doi.org/10.1097/GRF.0b013e3182a9ece0

[8] Lewiecki, E.M., Adler, R.A., Bilezikian, J.P., Bouxsein, M.L., Marcus, R., McClung, M.R., et al. (2013) Osteoporosis Update from the 2012 Santa Fe Bone Symposium. Journal of Clinical Densitometry, 16, 584-600. http://dx.doi.org/10.1016/j.jocd.2013.01.001

[9] Haymann, J.-P. (2010) Bone Densitometry: Principles and Indications. In ProgUrol - FMC, 20, F119-F122.

[10] El Maghraoui, A. (2004) Glucocorticoid-Induced Osteoporosis. Medical Press, 33, 12131217.

[11] Achemlal, L., Guerboub, A., Mounach, A., Nouijai, A., Ghazi, M. and Bezza, A., et al. (2006) 
Obesity Is a Protective Factor for Osteoporosis in Elderly Normal Moroccan Women. 19th Cong FRENCH Rhumatol CNIT, Paris, 3-6 December 2006, 1154.

[12] Briot, K., Cortet, B., Roux, C., Fardet, L., Abitbol, V., Bacchetta, J., et al. (2014) Update 2014 Recommendations on the Prevention and Treatment of Glucocorticoid-Induced Osteoporosis. Revue du Rhumatisme, 81, 385-394. http://dx.doi.org/10.1016/j.rhum.2014.07.009

[13] Bousson, V., Bergot, C., Sutter, B., Levitz, P. and Cortet, B., Scientific Committee of the Group for Research and Information on Osteoporosis (2012) Trabecular Bone Score (TBS): Available Knowledge, Clinical Relevance, and Future Prospects. Osteoporosis International, 23, 1489-1501. http://dx.doi.org/10.1007/s00198-011-1824-6

[14] Issever, A.S. and Link, T.M. (2011) Radiological Diagnosis of Osteoporosis. Zeitschrift Fur Rheumatologie, 70, 135-144, 145 quiz.

[15] Guglielmi, G., Damilakis, J., Solomou, G. and Bazzocchi, A. (2012) Quality Assurance of Imaging Tech Lunches Used in the Clinical Management of Osteoporosis. La Radiologia Medica (Torino), 117, 1347-1354. http://dx.doi.org/10.1007/s11547-012-0881-z

Submit or recommend next manuscript to SCIRP and we will provide best service for you:

Accepting pre-submission inquiries through Email, Facebook, LinkedIn, Twitter, etc. A wide selection of journals (inclusive of 9 subjects, more than 200 journals)

Providing 24-hour high-quality service

User-friendly online submission system

Fair and swift peer-review system

Efficient typesetting and proofreading procedure

Display of the result of downloads and visits, as well as the number of cited articles

Maximum dissemination of your research work

Submit your manuscript at: http://papersubmission.scirp.org/

Or contact ojra@scirp.org 